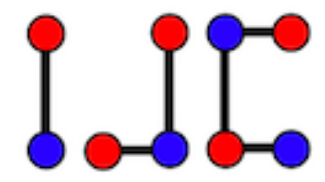

\title{
Laplacian energy of trees with at most 10 vertices
}

\author{
Masood Ur Rehman ${ }^{\mathrm{a}}$, Muhammad Ajmal ${ }^{\mathrm{a}}$, Tayyab Kamran ${ }^{\mathrm{b}}$ \\ ${ }^{a}$ School Of Mathematical Sciences, University of Science and Technology of China, Hefei, Anhui, 230026, P.R. China \\ ${ }^{b}$ Department of Mathematics, Quaid-I-Azam University, Islamabad-Pakistan \\ masood@mail.ustc.edu.cn,majmal@mail.ustc.edu.cn, tayyabkamran@gmail.com
}

\begin{abstract}
Let $T_{n}$ be the set of all trees with $n \leq 10$ vertices. We show that the Laplacian energy of any tree $T_{n}$ is strictly between the Laplacian energy of the path $P_{n}$ and the star $S_{n}$, the authors partially proving that the conjecture hold for any tree $T_{n}$, where $n \leq 10$.
\end{abstract}

Keywords: Tree, Laplacian energy

Mathematics Subject Classification: 05C50

DOI: $10.19184 /$ ijc.2018.2.1.3

\section{Introduction and preliminaries}

Let $G=(V, E)$ be a finite, simple and undirected graph with vertices $V=\{1,2, \ldots, n\}$ and $m=|E|$ edges. The degree of a vertex $u \in V$ will be denoted by $d_{n}$. Let $G$ have adjacency matrix $A$ with eigenvalues $\lambda_{1} \geq \lambda_{2} \geq \ldots \geq \lambda_{n}$, and the Laplacian matrix $L=D-A$, where $D$ is the diagonal matrix of vertex degrees, with eigenvalues $\mu_{1} \geq \mu_{2} \geq \ldots \geq \mu_{n}=0$. Additional details on the theory of graph spectra may be found in [1].

The energy and Laplacian energy of $G$ are defined as follows

$$
E(G)=\sum_{i=1}^{n}\left|\lambda_{i}\right|, \quad L E(G)=\sum_{i=1}^{n}\left|\mu_{i}-\frac{2 m}{n}\right| .
$$

The energy of a graph was defined by Ivan Gutman in [2] and it has a long known chemical applications; for details see surveys $[3,4]$. On the other hand, the Laplacian energy of a graph $G$,

Received: 13 Oct 2017, Revised: 09 Mar 2018, Accepted: 11 Apr 2018. 
defined by Ivan Gutman and Zhou in [5].

An important problem in the area of spectral graph theory is to determine which graph $G$ among all graphs with $n$ vertices has the maximum energy $E(G)$. Among the trees (a tree $T$ is a connected undirected graph without cycles), it has been long known [7] that for positive integer $n$, the path $P_{n}$ has maximum energy and the star $S_{n}$ has minimum energy. For the Laplacian energy, few results are established, so that the extremal energy graphs are not known even for trees. In [8], Radenković and Gutman studied the correlation between the energy and Laplacian energy of trees. In that paper, the energy and the Laplacian energy for trees are computed. They found that the energy and the Laplacian energy of a tree are inversely proportional, and formulated the following conjecture:

Conjecture 1. Let $T_{n}$ be a tree on $n$ vertices. Then

$L E\left(P_{n}\right) \leq L E\left(T_{n}\right) \leq L E\left(S_{n}\right)$.

Furthermore, in [9], Trevisan et al.,(2011) showed that the above conjecture is true for the restricted class of trees, namely those whose diameter is 3. Our goal here is to show that the Conjecture 1.1, is also true for all trees with at most 10 vertices. The plan of the paper is as follows: In the next section we will first give a notation to name trees. Next, we establish a result (Lemma 2.1) about trees with at most 10 vertices, Theorem 2.2, gives the fact that the Conjecture 1.1 , is true for all trees with at most 10 vertices. We will conclude the paper with a discussion about conclusions and future work.

\section{Main results}

\subsubsection{Names of general trees}

In tables below, we need a notation to name trees. Given a tree, pick some vertex and call it the root. Now walk along the tree (depth-first), starting at the root, and when a vertex is encountered for the first time, write down its distance to the root. The sequence of integers obtained is called a level sequence for the tree. A tree is uniquely determined by any level sequence. The parent of a vertex labeled $m$ is the last vertex encountered earlier that was labeled $m-1$. For example, the tree $K_{1,9}$ gets level sequence 0111111111 if the vertex of degree 9 is chosen as root, 012222222 otherwise. We use exponent to indicate repetition: 0111111111 can be written $01^{9}$ and 0121212 as $0(12)^{3}$.

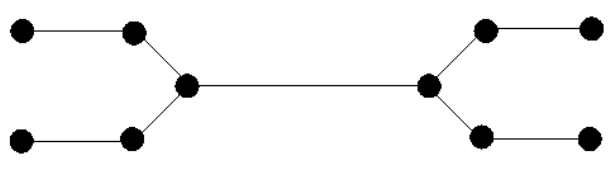

Figure 1. $0(12)^{2} 1(23)^{2}$

Lemma 2.1. There are exactly 199 trees with at most 10 vertices. 
Proof. If there is a tree with no edge, then it is a single vertex (it is $\# 0$ on the TABLE 1 ). Moreover, from the appendix (table\# 2) of [1], one can find all trees $T_{n}$ for $2 \leq n \leq 10$, where $n$ is the number of vertices in $T$.

Now we are ready to discuss the main result of the paper.

Theorem 2.1. The conjecture $L E\left(P_{n}\right) \leq L E\left(T_{n}\right) \leq L E\left(S_{n}\right)$ is true for $1 \leq n \leq 10$.

Proof. By Lemma 2.1, we have all trees with at most 10 vertices, we give all these trees in third column on the tables below in the form of level sequence. Now, from section 1 , we have $L=D-A$ the Laplacian matrix of $T$, and $L E(T)=\sum_{i=1}^{n}\left|\mu_{i}-\frac{2 m}{n}\right|$ is the Laplacian energy of $T$. By direct calculation (one can do this exercise by computer, by use of suitable mathematical softwares, for example Matlab or Mathematica) we find the Laplacian spectrum of $T$ (the set of eigenvalues of the matrix $L$, where the exponent of an eigenvalue denotes the multiplicity of the corresponding eigenvalue), and Laplacian energy of each $T$. Here, we note that on TABLE 1, TABLE 2, TABLE 3 and TABLE 4 we give all trees in such a way that the star $S_{n}$ appear first and the path $P_{n}$ appear last and all other trees appear between $S_{n}$ and $P_{n}$ for the same $n$.

TABLE 1, TABLE 2, TABLE 3 and TABLE 4 gives a serial number, the number of vertices $n$, a level sequence, the Laplacian spectrum and the Laplacian energy of $T$. For $n=1,2,3$ we can see from TABLE 1 , that $L E\left(P_{n}\right)=L E\left(T_{n}\right)=L E\left(S_{n}\right)$. For $4 \leq n \leq 10$ from TABle 1 , TABLE 2 , TABLE 3 and TABLE 4 it is easy to observe that $L E\left(P_{n}\right) \leq L E\left(T_{n}\right) \leq L E\left(S_{n}\right)$, with equality if and only if $P_{n}=T_{n}=S_{n}$.

\begin{tabular}{|c|c|c|c|c|}
\hline \# & $\mathrm{n}$ & trees & Laplacian spectrum & $\overline{L E\left(T_{n}\right)}$ \\
\hline 0 & 0 & 0 & 0 & 0 \\
\hline 1 & 2 & 01 & 0,2 & 2 \\
\hline 2 & 3 & $01^{2}$ & $0,1,3$ & 3.3333 \\
\hline 3 & 4 & $01^{3}$ & $0,1^{2}, 4$ & 5 \\
\hline 4 & 4 & 0112 & $0,0.5858,2,3.4142$ & 4.8284 \\
\hline 5 & 5 & $01^{4}$ & $0,1^{3}, 5$ & 6.8000 \\
\hline 6 & 5 & $01^{2} 12$ & $0,0.5188,1,2.3111,4.1701$ & 6.5624 \\
\hline 7 & 5 & $0(12)^{2}$ & $0,0.3820,1.3820,2.6180,3.6180$ & 6.0720 \\
\hline 8 & 6 & $01^{5}$ & $0,1^{4}, 6$ & 8.6667 \\
\hline 9 & 6 & $01^{3} 12$ & $0,0.4859,1^{2}, 2.4280,5.0861$ & 8.3615 \\
\hline 10 & 6 & $01^{2} 12^{2}$ & $0,0.4384,1^{2}, 3,4$ & 8.9616 \\
\hline 11 & 6 & $01^{2} 123$ & $0,0.3249,1,1.4608,3,4.2143$ & 7.7619 \\
\hline 12 & 6 & $01(12)^{2}$ & $0,0.3820,0.6972,2,2.6180,4.3028$ & 7.8416 \\
\hline 13 & 6 & 012123 & $0,0.2679,1,2,3,3.7321$ & 7.4642 \\
\hline
\end{tabular}


Table 1. (cont.)

\begin{tabular}{|c|c|c|c|c|}
\hline \# & $\mathrm{n}$ & trees & Laplacian spectrum & $\overline{L E\left(T_{n}\right)}$ \\
\hline 14 & 7 & $01^{6}$ & $0,1^{5}, 7$ & 10.5714 \\
\hline 15 & 7 & $01^{4} 12$ & $0,0.4659,1^{3}, 2.4827,6.0514$ & 10.2111 \\
\hline 16 & 7 & $01^{3} 12^{2}$ & $0,0.3983,1^{3}, 3.3399,5.2618$ & 10.3463 \\
\hline 17 & 7 & $01^{3} 123$ & $0,0.2955,1^{2}, 1.4911,3.1169,5.0965$ & 9.5697 \\
\hline 18 & 7 & $01^{2}(12)^{2}$ & $0,0.3820,0.6086,1,2.2271,2.6180,5.1642$ & 9.7330 \\
\hline 19 & 7 & $01^{2} 123^{2}$ & $0,0.2679,1^{2}, 1.5858,3.7321,4.4142$ & 9.4355 \\
\hline 20 & 7 & $01^{2} 1223$ & $0,0.3217,0.6802,1,2.1397,3.2297,4.6287$ & 9.7105 \\
\hline 21 & 7 & $01^{2} 1234$ & $0,0.2254,1^{2}, 2.1859,3.3604,4.2283$ & 9.2635 \\
\hline 22 & 7 & 0112123 & $0,0.2603,0.6262,1.4055,2.2742,3.0996,4.3342$ & 9.1303 \\
\hline 23 & 7 & $0(12)^{3}$ & $0,0.3820,0.3820,1.5858,2.6180^{2}, 4.4142$ & 9.0147 \\
\hline 24 & 7 & $0(123)^{2}$ & $0,0.1981,0.7530,1.5550,2.4450,3.2470,3.8019$ & 8.7021 \\
\hline 25 & 8 & $01^{7}$ & $0,1^{6}, 8$ & 12.5000 \\
\hline 26 & 8 & $01^{5} 12$ & $0,0.4525,1^{4}, 2.5135,7.0340$ & 12.0950 \\
\hline 27 & 8 & $01^{4} 12^{2}$ & $0,0.3738,1^{4}, 3.4849,6.1413$ & 12.2524 \\
\hline 28 & 8 & $01^{3} 12^{3}$ & $0,0.3542,1^{4}, 4,5.6458$ & 12.2616 \\
\hline 29 & 8 & $01^{4} 123$ & $0,0.2774,1^{4}, 3.1610,6.0548$ & 11.9384 \\
\hline 30 & 8 & $01^{3}(12)^{2}$ & $0,0.3820,0.5607,1^{2}, 2.3389,2.6180,6.1004$ & 11.6146 \\
\hline 31 & 8 & $01^{3} 123^{2}$ & $0,0.2384,1^{3}, 1.6367,4,5.1249$ & 11.2488 \\
\hline 32 & 8 & $01^{3} 1223$ & $0,0.2888,0.6742,1^{2}, 2.1694,3.5857,5.2819$ & 11.8740 \\
\hline 33 & 8 & $01^{2} 12^{2} 23$ & $0,0.3187,0.5858,1^{2}, 2.3579,3.4142,5.3234$ & 11.6910 \\
\hline 34 & 8 & $01^{3} 1234$ & $0,0.023,1^{3}, 2.2472,3.4527,5.0979$ & 11.0955 \\
\hline 35 & 8 & $01^{2} 12123$ & $0,0.2538,0.5472,1,1.4689,2.4066,3.1504,5.1732$ & 10.9603 \\
\hline 36 & 8 & $01(12)^{3}$ & $0,0.3820^{2}, 0.7639,2,2.6180,2.6180,5.2361$ & 10.9442 \\
\hline 37 & 8 & $012^{2} 123^{2}$ & $0,0.1864,1^{3}, 2.4707,4,4.3429$ & 11.1272 \\
\hline 38 & 8 & $012^{2} 1223$ & $0,0.2137,0.6177,1,1.4977,2.3537,3.8408,4.4763$ & 10.8417 \\
\hline 39 & 8 & $01^{2} 12234$ & $0,0.2243,0.5858,1,1.4108,2.7237,3.4142,4.6412$ & 11.0582 \\
\hline 40 & 8 & $01^{2} 1(23)^{2}$ & $0,0.3065,0.3820,1,1.6703,2.6180,3.3297,4.6935$ & 10.7824 \\
\hline 41 & 8 & 01121223 & $0,0.2509,0.5858,0.7287,2,2.3349,3.4142,4.6855$ & 10.8692 \\
\hline 42 & 8 & $01^{2} 12345$ & $0,0.1667,0.7276,1,1.6353,2.6729,3.5643,4.2332$ & 10.4408 \\
\hline 43 & 8 & $01(123)^{2}$ & $0,0.1981,0.4915,1.3204,1.5550,2.8258,3.2470,4.3623$ & 10.3701 \\
\hline 44 & 8 & 01121234 & $0,0.1864,0.5858,1,2,2.4707,3.4142,4.3429$ & 10.4556 \\
\hline 45 & 8 & $0(12)^{2} 123$ & $0,0.2434,0.3820,1.1798,2,2.6180,3.1386,4.4383$ & 10.3897 \\
\hline 46 & 8 & 01231234 & $0,0.1522,0.5858,1.2346,2,2.7654,3.4142,3.8478$ & 10.0548 \\
\hline 47 & 9 & $01^{8}$ & $0,1^{7}, 9$ & 14.4440 \\
\hline 48 & 9 & $01^{6} 12$ & $0,0.4428,1^{5}, 2.5330,8.0242$ & 14.0033 \\
\hline 49 & 9 & $01^{5} 12^{2}$ & $0,0.3572,1^{5}, 3.5554,7.0874$ & 14.1745 \\
\hline
\end{tabular}


Table 2

\begin{tabular}{|c|c|c|c|c|}
\hline \# & $\mathrm{n}$ & trees & Laplacian spectrum & $L E\left(T_{n}\right)$ \\
\hline 50 & 9 & $01^{5} 123$ & $0,0.2650,1^{5}, 3.1832,7.0355$ & 13.8426 \\
\hline 51 & 9 & $01^{4}(12)^{2}$ & $0,0.3820,0.5300,1^{3}, 2.4027,2.6180,7.0672$ & 13.5092 \\
\hline 52 & 9 & $01^{4} 12^{3}$ & $0,0.32721^{5}, 4.3519,6.3209$ & 14.2345 \\
\hline 53 & 9 & $01^{4} 123^{2}$ & $0,0.2201,1^{4}, 1.6634,4.0549,6.0615$ & 13.1218 \\
\hline 54 & 9 & $01^{4} 1223$ & $0,0.2679,0.67111^{3}, 2.1814,3.7321,6.1474$ & 13.4552 \\
\hline 55 & 9 & $01^{3} 1212^{2}$ & $0,0.3158,0.5356,1^{3}, 2.4475,3.5152,6.1860$ & 13.6302 \\
\hline 56 & 9 & $0\left(12^{3}\right)^{2}$ & $0,0.2087,1^{4} 1.6972,4.7913,5.3028$ & 13.0771 \\
\hline 57 & 9 & $01^{4} 1234$ & $0,0.1876,1^{4}, 2.2755,3.4819,6.0550$ & 12.9581 \\
\hline 58 & 9 & $01^{3} 12^{2} 23$ & $0,0.2825,1^{3}, 2.3735,4.0864,5.6793$ & 13.6116 \\
\hline 59 & 9 & $01^{3} 12123$ & $0,0.2483,0.5063,1^{2}, 1.4950,2.4702,3.1767,6.1036$ & 12.8342 \\
\hline 60 & 9 & $01^{2}(12)^{3}$ & $0,0.3820^{2}, 0.6711,1,2.1814,2.6180^{2}, 6.1474$ & 12.9075 \\
\hline 61 & 9 & $0112^{2} 12^{2}$ & $0,0.2377,0.6484,1^{3}, 2.6501,4.3124,5.3314$ & 13.5611 \\
\hline 62 & 9 & $01^{2}\left(12^{2}\right)^{2}$ & $0,0.2679,0.5505,1^{3}, 3,3.7321,5.4495$ & 13.6965 \\
\hline 63 & 9 & $012^{2} 123^{3}$ & $0,0.1649,1^{4}, 2.5680,4.1652,5.1019$ & 13.0035 \\
\hline 64 & 9 & $0122312^{3}$ & $0,0.1884,0.6144,1^{2}, 1.5333,2.3798,4.1545,5.1296$ & 12.6611 \\
\hline 65 & 9 & $012^{2} 12^{2} 23$ & $0,0.2043,0.5405,1^{2}, 1.5989,2.4425,4.0170,5.1969$ & 12.6460 \\
\hline 66 & 9 & $0112312^{3}$ & $0,0.2022,0.5693,1^{2}, 1.4124,2.8273,3.7046,5.2842$ & 13.0304 \\
\hline 67 & 9 & $0(12)^{2} 12^{3}$ & $0,0.2679,0.3820,1^{2}, 1.6972,2.6180,3.7321,5.3028$ & 12.6391 \\
\hline 68 & 9 & $01^{2} 12^{2} 123$ & $0,0.2232,0.4919,1^{2}, 1.4712,3,3.4838,5.3298$ & 12.9606 \\
\hline 69 & 9 & $01^{2} 121223$ & $0,0.2427,0.5371,0.6893,1,2.1297,2.4166,3.6434,5.3411$ & 12.8395 \\
\hline 70 & 9 & $01(12)^{2} 12^{2}$ & $0,0.3047,0.3820,0.7566,1,2.0960,2.6180,3.4609,5.3818$ & 12.8912 \\
\hline 71 & 9 & $01^{3} 1234$ & $0,0.1487,0.7169,1^{2}, 1.6629,2.7405,3.6330,5.0980$ & 12.2763 \\
\hline 72 & 9 & $01^{2} 12334^{2}$ & $0,0.1830,0.5723,1^{2}, 1.5095,3,4.0444,4.6907$ & 12.8036 \\
\hline 73 & 9 & $01^{2} 12323^{2}$ & $0,0.2679,0.3446,1^{2}, 1.7892,3,3.7321,4.8662$ & 12.5528 \\
\hline 74 & 9 & $01^{2}(123)^{2}$ & $0,0.1981,0.4116,1,1.4064,1.5550,3,3.2470,5.1819$ & 12.1929 \\
\hline 75 & 9 & $01122312^{2}$ & $0,0.2118,0.5546,0.7223,1,2.0782,2.7338,3.8525,4.8468$ & 12.8004 \\
\hline 76 & 9 & $01^{2} 121234$ & $0,0.1774,0.5242,1^{2}, 2.1609,2.4961,3.4670,5.1743$ & 12.3745 \\
\hline 77 & 9 & $01(12)^{2} 123$ & $0,0.2398,0.3820,0.7199,1.4240,2.2032,2.6180,3.1692,5.2439$ & 12.2464 \\
\hline 78 & 9 & $0(12)^{4}$ & $0,0.3820,0.3820,0.3820,1.6972,2.6180,2.6180^{2}, 5.3028$ & 12.0914 \\
\hline 79 & 9 & $0\left(123^{2}\right)^{2}$ & $0,0.1392,0.6972,1^{2}, 1.7459,3,4.1149,4.3028$ & 12.1887 \\
\hline 80 & 9 & $01223412^{2}$ & $0,0.1658,0.4679,1,1.3434,1.6527,3,3.8794,4.4909$ & 12.0738 \\
\hline 81 & 9 & $01223123^{2}$ & $0,0.1538,0.5764,1^{2}, 2.1128,2.6757,4.0748,4.4065$ & 12.3174 \\
\hline 82 & 9 & $01123412^{2}$ & $0,0.1627,0.5321,1^{2}, 2.0892,3,3.5723,4.6437$ & 12.3882 \\
\hline 83 & 9 & $012^{2} 1(23)^{2}$ & $0,0.1953,0.3820,1,1.2108,2.1449,2.6180,3.9064,4.5426$ & 12.2016 \\
\hline 84 & 9 & $0(1223)^{2}$ & $0,0.1729,0.5587,0.6617,1.4331,2.2091,2.4851,3.9563,4.5231$ & 12.1250 \\
\hline 85 & 9 & $012^{2} 12123$ & $0,0.2217,0.3327,1,1.1923,2.1071,3,3.4413,4.7049$ & 12.2844 \\
\hline 86 & 9 & 0112312123 & $0,0.1862,0.4822,0.7043,1.4073,2.1338,2.8532,3.5372,4.6958$ & 12.2178 \\
\hline 87 & 9 & $01121(23)^{2}$ & $0,0.2311,0.3820,0.6416,1.6129,2.2591,2.6180,3.5132,4.7421$ & 12.0426 \\
\hline 88 & 9 & $01^{2} 123456$ & $0,0.1289,0.5540,1,1.2613,2.1326,3,3.6881,4.2350$ & 11.8899 \\
\hline
\end{tabular}


Table 3.

\begin{tabular}{|c|c|c|c|c|}
\hline \# & $\mathrm{n}$ & trees & Laplacian spectrum & $L E\left(T_{n}\right)$ \\
\hline 89 & 9 & 011231234 & $0,0.1506,0.4266,1,1.4229,2.1724,3,3.4576,4.3699$ & 11.7776 \\
\hline 90 & 9 & 011212345 & $0,0.1404,0.5362,0.7754,1.5803,2.2449,2.7784,3.5988,4.3455$ & 11.7131 \\
\hline 91 & 9 & $0(123)^{2} 12$ & $0,0.1981,0.3004,1,1.5550,2.2391,3,3.2470,4.4605$ & 11.6709 \\
\hline 92 & 9 & $0(12)^{2} 1234$ & $0,0.1708,0.3820,0.8503,1.6761,2.4165,2.6180,3.4421,4.4442$ & 11.6194 \\
\hline 93 & 9 & $0(1234)^{2}$ & $0,0.1206,0.4679,1,1.6527,2.3473,3,3.5321,3.8794$ & 11.2954 \\
\hline 94 & 10 & $01^{9}$ & $0,1^{8}, 10$ & 16.4000 \\
\hline 95 & 10 & $01^{7} 12$ & $0,0.4355,1^{6}, 2.5464,9.0181$ & 15.9290 \\
\hline 96 & 10 & $01^{6} 12^{2}$ & $0,0.3451,1^{6}, 3.5956,8.0593$ & 16.1098 \\
\hline 97 & 10 & $01^{6} 123$ & $0,0.2560,1^{5}, 1.5227,3.1964,8.0249$ & 15.2426 \\
\hline 98 & 10 & $01^{5}(12)^{2}$ & $0,0.3820,0.5085,1^{4}, 2.4434,2.6180,8.0480$ & 15.4189 \\
\hline 99 & 10 & $01^{5} 12^{3}$ & $0,0.3087,1^{6}, 4.5111,7.1801$ & 16.1825 \\
\hline 100 & 10 & $01^{4} 12^{4}$ & $0,0.2984,1^{6}, 5,6.7016$ & 16.2032 \\
\hline 101 & 10 & $01^{5} 123^{2}$ & $0,0.2076,1^{5}, 1.6797,4.0748,7.0378$ & 15.0253 \\
\hline 102 & 10 & $01^{5} 1223$ & $0,0.2535,0.6693,1^{4}, 2.1879,3.7996,7.0897$ & 15.3544 \\
\hline 103 & 10 & $01^{4} 1212^{2}$ & $0,0.3130,0.5041,1^{4}, 2.4928,3.5709,7.1192$ & 15.5658 \\
\hline 104 & 10 & $01^{5} 1234$ & $0,0.1775,1^{5}, 2.2914,3.495621,7.0355$ & 14.8450 \\
\hline 105 & 10 & $01^{4} 12123$ & $0,0.2436,0.4813,1^{3}, 1.5090,2.5055,3.1921,7.0686$ & 14.7115 \\
\hline 106 & 10 & $01^{3}(12)^{3}$ & $0,0.3820^{2}, 0.6168,1^{2} 2.2835,2.6180^{2}, 7.0996$ & 14.8383 \\
\hline 107 & 10 & $01^{4} 123^{3}$ & $0,0.1902,1^{5}, 1.7292,5,6.0806$ & 14.9612 \\
\hline 108 & 10 & $01^{4} 12^{2} 23$ & $0,0.2592,0.5749,1^{4}, 2.3791,4.4541,6.3327$ & 15.5318 \\
\hline 109 & 10 & $01^{3} 1212^{3}$ & $0,0.2767,0.5283,1^{4}, 2.4558,4.3827,6.3565$ & 15.5900 \\
\hline 110 & 10 & $01^{4} 1223^{2}$ & $0,0.2183,0.6439,1^{4}, 2.6982,4.2803,6.1592$ & 15.4755 \\
\hline 111 & 10 & $01^{3}\left(12^{2}\right)^{2}$ & $0,0.2679,0.4921,1^{4}, 3.2444,3.7321,6.2635$ & 15.6800 \\
\hline 112 & 10 & $01^{4} 1234^{2}$ & $0,0.1512,1^{5}, 2.6112,4.1820,6.0555$ & 14.8975 \\
\hline 113 & 10 & $01\left(12^{3}\right)^{2}$ & $0,0.2087,0.6385,1^{4}, 2.8326,4.7913,5.5289$ & 15.5056 \\
\hline 114 & 10 & $01^{4} 12334$ & $0,0.1723,0.6127,1^{4}, 2.3922,4.2090,6.0621$ & 15.0783 \\
\hline 115 & 10 & $01^{2} 12334$ & $0,0.1967,0.5011,1^{3}, 1.6429,2.4895,4.0600,6.1098$ & 14.5186 \\
\hline 116 & 10 & $01^{3} 12^{2} 23^{2}$ & $0,0.2370,0.5379,1^{4}, 3.1628,4.3148,5.7475$ & 15.6502 \\
\hline 117 & 10 & $01^{4} 12234$ & $0,0.1875,0.5604,1^{3}, 1.6972,1.4131,2.8596,3.8315,6.1478$ & 14.8779 \\
\hline 118 & 10 & $01^{4} 1(23)^{2}$ & $0,0.2442,0.3820,1^{3}, 1.7101,2.6180,3.8920,6.1537$ & 14.5274 \\
\hline 119 & 10 & $012^{2} 1^{3} 123$ & $0,0.2222,0.44121,1^{3}, 1.4963,3.1080,3.5435,6.1888$ & 14.8806 \\
\hline 120 & 10 & $01^{3} 121223$ & $0,0.2357,0.5027,0.6782,1^{2}, 2.1648,2.4725,3.7543,6.1917$ & 14.7667 \\
\hline 121 & 10 & $012^{2} 1^{1}(12)^{2}$ & $0,0.3030,0.3820,0.6596,1^{2}, 2.2704,2.6180,3.5379,6.2291$ & 14.9108 \\
\hline 122 & 10 & $012^{2} 123^{3}$ & $0,0.1442,1^{5}, 2.6784,5,5.1774$ & 14.9116 \\
\hline 123 & 10 & $012^{3} 12^{2} 23$ & $0,0.1775,0.5379,1^{3}, 1.6516,2.4544,4.8393,5.3393$ & 14.4660 \\
\hline 124 & 10 & $01^{4} 12345$ & $0,0.1370,0.7110,1^{3}, 1.6764,2.7685,3.6520,6.0550$ & 14.1511 \\
\hline 125 & 10 & $01^{3} 12^{2} 234$ & $0,0.2022,0.4696,1^{3}, 1.4719,3.0620,4.1122,5.6821$ & 15.6821 \\
\hline 126 & 10 & $01^{2} 1(123)^{2}$ & $0,0.1981,0.3676,1^{2}, 1.4469,1.5550,3.0787,3.2470,6.1068$ & 14.0649 \\
\hline 127 & 10 & $01^{2} 1121234$ & $0,0.1704,0.4911,1^{3}, 2.2293,2.5173,3.4880,6.1038$ & 14.2769 \\
\hline
\end{tabular}


Table 4

\begin{tabular}{|c|c|c|c|c|}
\hline \# & $\mathrm{n}$ & trees & Laplacian spectrum & $L E\left(T_{n}\right)$ \\
\hline 128 & 10 & $01^{3} 12(23)^{2}$ & $0,0.2942,0.3820,0.7541,1^{2}, 2.1157,2.6180,4.1533,5.7127$ & 14.7994 \\
\hline 129 & 10 & $01^{2} 1212^{2} 23$ & $0,0.2330,0.5188,0.6158,1^{2}, 2.3111,2.4408,4.1701,5.7105$ & 14.8649 \\
\hline 130 & 10 & $01^{2}(12)^{2} 123$ & $0,0.2366,0.3820,0.6298,1,1.4757,2.3204,2.6180,3.1871,6.1504$ & 14.1518 \\
\hline 131 & 10 & $0(12)^{3} 112$ & $0,0.3820^{3}, 0.8074,2,2.6180^{3}, 6.1926$ & 14.0932 \\
\hline 132 & 10 & $012^{3} 1223^{2}$ & $0,0.1600,0.5669,1^{3}, 1.5488,3.0612,4.5184,5.1446$ & 14.6485 \\
\hline 133 & 10 & $0112^{3} 123^{2}$ & $0,0.1640,0.5531,1^{3}, 1.5129,3.2827,4.1972,5.2902$ & 14.7401 \\
\hline 134 & 10 & $01^{2} 12^{2} 123^{2}$ & $0,0.1800,0.4792,1^{3}, 1.6050,3.3220,4.0680,5.3458$ & 14.6716 \\
\hline 135 & 10 & $012^{3} 1212^{2}$ & $0,0.2357,0.3380,1^{3}, 1.8265,3.0847,4.1617,5.3534$ & 14.4526 \\
\hline 136 & 10 & $0112^{3} 1223$ & $0,0.1881,0.5415,0.7203,1^{2}, 2.0898,2.8800,4.2424,5.3379$ & 14.7002 \\
\hline 137 & 10 & $01^{2} 121223^{2}$ & $0,0.2015,0.5188,0.6721,1^{2}, 2.3111,2.7424,4.1701,5.3839$ & 14.8151 \\
\hline 138 & 10 & $01^{2} 12^{2} 1223$ & $0,0.2100,0.4862,0.6872,1^{2}, 2.1532,3.1369,3.8639,5.4627$ & 14.8333 \\
\hline 139 & 10 & $0112\left(12^{2}\right)^{2}$ & $0,0.2679,0.3426,0.7482,1^{2}, 2.2345,3.1746,3.7321,5.5002$ & 14.8838 \\
\hline 140 & 10 & $0\left(123^{2}\right)^{2}$ & $0,0.1231,0.6842,1^{3}, 1.7846,3.0971,4.2124,5.0986$ & 14.0162 \\
\hline 141 & 10 & $012^{3} 12234$ & $0,0.1466,0.4582,1^{2}, 1.3494,1.6947,3.0251,4.1959,5.1302$ & 13.9023 \\
\hline 142 & 10 & $012^{3} 12334$ & $0,0.1353,0.5723,1^{3}, 2.1398,2.7545,4.2955,5.1026$ & 14.1848 \\
\hline 143 & 10 & $012^{2} 12^{2} 234$ & $0,0.1648,0.3883,1^{2}, 1.4358,1.6684,3.1150,4.0226,5.2050$ & 13.8853 \\
\hline 144 & 10 & $012^{3} 1(23)^{2}$ & $0,0.1695,0.3820,1^{2}, 1.2228,2.1971,2.6180,4.2756,5.1350$ & 14.0 \\
\hline 145 & 10 & $012^{2} 123^{2} 34$ & $0,0.1442,0.5188,1^{3}, 2.3111,2.6784,4.1701,5.1774$ & 14.2740 \\
\hline 146 & 10 & $0\left(12^{2}\right)^{3}$ & $0,0.2679^{2}, 1^{3}, 2$ & 14.5284 \\
\hline 147 & 10 & $01^{3} 122345$ & $0,0.1473,0.5085,1^{3}, 2.1053,3.1771,3.7773,5.2844$ & 14.2883 \\
\hline 148 & 10 & $0112^{2} 1223^{2}$ & $0,0.1792,0.5188,0.7141,1^{2}, 2.3111,3.1593,4.1701,4.9474$ & 14.7158 \\
\hline 149 & 10 & $012^{2} 231223$ & $0,0.1626,0.5188,0.6270,1,1.5072,2.3111,2.5027,4.1701,5.2005$ & 14.7158 \\
\hline 150 & 10 & $012^{2} 1^{2} 1234$ & $0,0.1592,0.4563,1^{3}, 2.2121,3.2583,3.5836,5.3305$ & 14.3690 \\
\hline 151 & 10 & $012^{2} 12(23)^{2}$ & $0,0.1898,0.3820,0.7154,1,1.5268,2.2743,2.6180,4.0296,5.2641$ & 13.9720 \\
\hline 152 & 10 & $01^{2} 1231223$ & $0,0.1861,0.4111,0.6824,1,1.4697,2.1671,3.0584,3.6781,5.3472$ & 14.1015 \\
\hline 153 & 10 & $0112^{2} 23123$ & $0,0.1769,0.4716,0.6288,1,1.4112,2.3497,2.8697,3.7491,5.3430$ & 14.2230 \\
\hline 154 & 10 & $012^{2} 112123$ & $0,0.2207,0.3298,0.7073,1,1.4285,2.3268,3.0917,3.5074,5.3876$ & 14.2272 \\
\hline 155 & 10 & $01^{2} 121(23)^{2}$ & $0,0.2206,0.3820,0.5548,1,1.6771,2.4011,2.6180,3.7871,5.3595$ & 13.9312 \\
\hline 156 & 10 & $011212(23)^{2}$ & $0,0.2263,0.3820,0.6274,0.7726,2,2.2925,2.6180,3.6837,5.3975$ & 13.9834 \\
\hline 157 & 10 & $012^{2}(12)^{3}$ & $0,0.2971,0.3820^{2}, 1,1.7713,2.6180,2.6180,3.4942,5.4374$ & 13.9352 \\
\hline 158 & 10 & $01\left(123^{2}\right)^{2}$ & $0,0.1392,0.4384,1^{2}, 1.3820,1.7459,3.6180,4.1149,4.5616$ & 13.7890 \\
\hline 159 & 10 & $01^{3} 123456$ & $0,0.1148,0.5399,1^{2}, 1.2709,2.1739,3.0636,3.7387,5.0981$ & 13.7487 \\
\hline 160 & 10 & $01^{2} 123445^{2}$ & $0,0.1338,0.5188,1^{3}, 2.3111,3.2108,4.1701,4.6554$ & 14.2948 \\
\hline 161 & 10 & $012123^{2} 12^{2}$ & $0,0.1772,0.3300,1^{2}, 1.2293,2.2486,3.2174,4.0502,4.7472$ & 14.1269 \\
\hline 162 & 10 & $01123^{2} 1223$ & $0,0.1535,0.4616,0.7026,1,1.5019,2.1589,3.2036,4.0827,4.7351$ & 13.9607 \\
\hline 163 & 10 & $012231223^{2}$ & $0,0.1487,0.5188,0.6496,1,1.4400,2.3111,3.0561,4.1701,4.7056$ & 14.0858 \\
\hline 164 & 10 & $0\left(12^{2}\right)^{2} 123$ & $0,0.2154,0.2679,1^{2}, 1.2059,2.3671,3.3375,3.7321,4.8742$ & \\
\hline 165 & 10 & & $.3621,1^{2}, 1.4749,2.2$ & \\
\hline
\end{tabular}


Table 4. (cont.)

\begin{tabular}{|c|c|c|c|c|}
\hline \# & $\mathrm{n}$ & trees & Laplacian spectrum & $L E\left(T_{n}\right)$ \\
\hline 166 & 10 & $0112^{2} 12234$ & $0,0.1614,0.4439,0.6905,1,1.4077,2.4604,3.083,3.9006,4.8522$ & 14.1930 \\
\hline 167 & 10 & $01^{2} 1212345$ & $0,0.1317,0.5006,0.7370,1,1.6424,2.3851,2.7880,3.6407,5.1744$ & 13.5765 \\
\hline 168 & 10 & $012^{2} 121223$ & $0,0.2076,0.3326,0.6394,1,1.7049,2.2883,3.0761,3.8552,4.8958$ & 13.8309 \\
\hline 169 & 10 & $011(23)^{2} 12^{2}$ & $0,0.1920,0.3820,0.6047,1,1.6249,2.6180,2.7557,3.9407,4.8821$ & 13.9926 \\
\hline 170 & 10 & 0112231223 & $0,0.1729,0.4755,0.6617,0.7420,2,2.2091,2.9065,3.9563,4.8760$ & 13.8958 \\
\hline 171 & 10 & $0112(123)^{2}$ & $0,0.1981,0.2937,0.6603,1.3427,1.5550,2.3831,3.0686,3.2470,5.2516$ & 13.5005 \\
\hline 172 & 10 & $01(12)^{2} 1234$ & $0,0.1655,0.3820,0.6815,1,2,2.4314,2.6180,3.4768,5.2448$ & 13.5420 \\
\hline 173 & 10 & $0(12)^{3} 123$ & $0,0.2318,0.3820^{2}, 1.2769,2,2.6180^{2}, 3.1815,5.3098$ & 13.4546 \\
\hline 174 & 10 & $0123^{2} 1234^{2}$ & $0,0.1088,0.5188,1^{2}, 1.2954,2.3111,3.3174,4.1701,4.2784$ & 13.7540 \\
\hline 175 & 10 & $012234512^{2}$ & $0,0.1288,0.3924,1^{2}, 1.5222,2.2184,3.3439,3.9000,4.4944$ & 13.5133 \\
\hline 176 & 10 & $011231234^{2}$ & $0,0.1257,0.4097,1^{2}, 1.4295,2.4234,3.0954,4.0925,4.4238$ & 13.6702 \\
\hline 177 & 10 & $012334123^{2}$ & $0,0.1172,0.5188,0.7586,1,1.6674,2.3111,3.0846,4.1701,4.3721$ & 13.4759 \\
\hline 178 & 10 & $012323412^{2}$ & $0,0.1640,0.2885,1^{2}, 1.6385,2.3252,3.0979,3.9293,4.5566$ & 13.4180 \\
\hline 179 & 10 & $011234512^{2}$ & $0,0.1236,0.4790,0.7723,1,1.5904,2.5350,3.1669,3.6885,4.6442$ & 13.6693 \\
\hline 180 & 10 & 0122341223 & $0,0.1378,0.4258,0.6323,1.3282,1.5820,2.34353 .0242,3.9923$ & 13.3879 \\
\hline 181 & 10 & $0(123)^{2} 123^{2}$ & $0,0.1392,0.3820,0.8299,1,1.7459,2.6180,2.6889,4.1149,4.4812$ & 13.4060 \\
\hline 182 & 10 & 0122312334 & $0,0.1277,0.5188,0.6297,1,2,2.3111,2.7968,4.1701,4.4458$ & 13.4476 \\
\hline 183 & 10 & $0(123)^{2} 12^{2}$ & $0,0.1981,0.2375,1^{2}, 1.5550,2.5634,3.2470,3.4832,4.7159$ & 13.6189 \\
\hline 184 & 10 & 0112312234 & $0,0.1487,0.3820,0.6496,1.3820,1.4400,2.6180,3.0561,3.6180,4.7056$ & 13.5954 \\
\hline 185 & 10 & $012123412^{2}$ & $0,0.1566,0.3280,0.8452,1,1.7534,2.4520,3.1820,3.5756,4.7070$ & 13.4334 \\
\hline 186 & 10 & 0122334123 & $0,0.1398,0.4249,0.6932,1,2,2.2574,3.1456,3.6414,4.6978$ & 13.4843 \\
\hline 187 & 10 & $01(23)^{2} 1223$ & $0,0.1561,0.3820,0.5965,1.1864,2,2.4539,2.6180,4.0305,4.5767$ & 13.3581 \\
\hline 188 & 10 & $0(12)^{2} 12234$ & $0,0.1700,0.3820,0.5078,1.3820,1.6959,2.6180,2.8758,3.6180,4.7505$ & 13.3246 \\
\hline 189 & 10 & 0121231223 & $0,0.1859,0.2989,0.6329,1.1826,2,2.3183,3.0437,3.5861,4.7517$ & 13.3995 \\
\hline 190 & 10 & $0(12)^{2} 1(23)^{2}$ & $0,0.2087,0.3820^{2}, 2,2.6180,2.6180,3.6180,4.7913$ & 13.2906 \\
\hline 191 & 10 & $01^{2} 1234567$ & $0,0.1029,0.4363,1^{2}, 1.7250,2.5064,3.2255,3.7678,4.2357$ & 13.0708 \\
\hline 192 & 10 & 0112312345 & $0,0.1172,0.3820,0.7586,1.3820,1.6674,2.6180,3.0846,3.6180,4.3721$ & 12.9855 \\
\hline 193 & 10 & $01(1234)^{2}$ & $0,0.1206,0.3489,1^{2}, 2,2.3473,3.2739,3.5321,4.3772$ & 13.0610 \\
\hline 194 & 10 & 0112123456 & $0,0.1100,0.4616,0.6697,1.2415,2,2.4010,3.0579,3.7120,4.3463$ & 13.0344 \\
\hline 195 & 10 & $0(123)^{3}$ & $0,0.1981^{2}, 0.8299,1.5550^{2}, 2.6889,3.2470^{2}, 4.4812$ & 12.9280 \\
\hline 196 & 10 & 0121231234 & $0,0.1479,0.2814,0.7873,1.2931,2,2.4631,3.0926,3.4687,4.4659$ & 12.9806 \\
\hline 197 & 10 & $0(12)^{2} 12345$ & $0,0.1277,0.3820,0.6297,1.3820,2,2.6180,2.7968,3.6180,4.4458$ & 12.9572 \\
\hline 198 & 10 & 0123412345 & $0,0.0979,0.3820,0.8244,1.3820,2,2.6180,3.1756,3.6180,3.9021$ & 12.6274 \\
\hline
\end{tabular}




\section{Discussion and concluding remarks}

In this paper, our attention was focused on the Laplacian energy of trees and on the partial proof of the Conjecture 1.1. We have shown that Laplacian energy of any tree $T_{n}$, with $n \leq 10$ vertices is strictly between the Laplacian energy of the path $P_{n}$ and the Laplacian energy of the star $S_{n}$, a step towards the proof of the Conjecture 1.1 .

\section{Future work}

let $S_{1, p}(p \geq 1)$ and $S_{1, q}(q \geq 1)$ are two stars, we introduced a special tree, denoted by $\mathbf{T}(p, q)$ by identifying one pendent vertex of $S_{1, p}$ and one pendent vertex of $S_{1, q}$, now we see that $\mathbf{T}(p, q)$ has diameter 4, with $n=p+q+3$ vertices; see the graph in FIGURE 2. We plan to show that the Conjecture 1.1, is hold for any tree $T_{n}$ such that $T_{n} \cong \mathbf{T}(p, q)$.

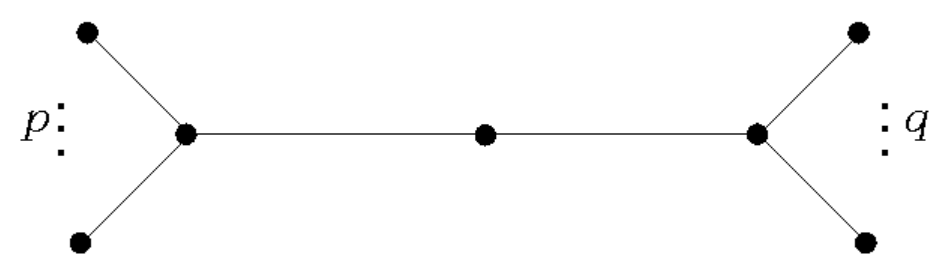

Figure 2. The graph $\mathbf{T}(p, q)$

Moreover, from [10, p. 69-83] we find all trees $T_{n}$ where $n \in\{11,12\}$, there are total 785 trees $T_{n}$ for which $n \in\{11,12\}$. We also plan to show that the Conjecture 1.1 , is hold for any tree $T_{n}$ where $n \in\{11,12\}$.

\section{Acknowledgements}

The first and second author is supported by the Chinese Scholarship Council at USTC, China. The authors would like to express their sincere gratitude to the referee for a very careful reading of this paper and for all his/her insightful comments. Which lead a number of improvements to this paper.

\section{References}

[1] D. M. Cvetković, M. Doob and H. Sachs, Spectra of Graphs- Theory and Application, Vol. 87. Academic Press, 1980. 
[2] I. Gutman, The energy of a graph, Ber. Math.Statist. Sekt. Forschungsz. Graz, 103 (1978), $1-22$.

[3] I. Gtman, Total $\pi$-electron energy of benzenoid hydrocarbons, Topics Curr. Chem., 162 (1992), 29-63.

[4] I. Gutman, The energy of a graph: old and new results,in: A. Betten, A. Kohnert, R. Laue, A. Wassermann(Eds.), Algebraic Combinatorics and Applications, Springer-Verlag, Berlin, 2001, pp. 196-211.

[5] I. Gutman, B. Zhou, Laplacian energy of a graph, Linear Algebra Appl. 414 (2006), 29-37.

[6] D.M. Cardoso, D. Cvetković, P. Rowlinson, S. Simić, A sharp lower bound for the least eigenvalue of the signless Laplacian of a non-bipartite graph, Linear Algebra Appl. 429 (2008), 2770-2780.

[7] I. Gutman, Acyclic systems with extremal Hückel $\pi$-electron energy, Theor. Chim. Acta 45 (1977), 79-87.

[8] S. Radenković, I. Gutman, Total $\pi$-electron energy and Laplacian energy: how far the analogy goes? J. Serb. Chem. Soc. 72(12) (2007) 1343-1350.

[9] V. Trevisan, J. B. Carvalho, Renata R. Del Vecchio, Cybele T.M. Vinagre, Laplacians energy of diameter 3 trees, Appl. Math. Lett., 24 (2011) 918-923.

[10] R. C. Read, R. J. Wilson, An Atlas of Graphs, The Clarendon Press Oxford University Press, NewYork, 1998.

[11] D. Stevanović, I. Gutman, M. U. Rehman, On spectral radius and energy of complete multipartite graphs, Ars Mathematica Contemporanea 9 (2015), 109-113. 